\title{
Data-driven Green's function retrieval from reflection data: Theory and example
}

Kees Wapenaar, Evert Slob, Filippo Broggini, Roel Snieder, Jan Thorbecke and Joost van der Neut

\section{summary}

Recently we introduced a new approach for retrieving the Green's response to a virtual source in the subsurface from reflection data at the surface. Unlike in seismic interferometry, no receiver is needed at the position of the virtual source. Here we present the theory behind this new method. First we introduce the Green's function $G$ and a so-called fundamental solution $F$ of an inhomogeneous medium. Next we derive a relation between $G$ and $F$, using reciprocity theorems. This relation is used as the basis for deriving a 3D single-sided Marchenko equation. We show that this equation is solved by a 3D autofocusing scheme and that the Green's function is obtained by combining the focusing wave field and its response in a specific way. We illustrate the method with a numerical example. 


\section{Introduction}

Recently we introduced a new approach for retrieving the Green's response to a virtual source in the subsurface from reflection data at the surface (Broggini and Snieder, 2012; Wapenaar et al., 2012a). Unlike in seismic interferometry, no receiver is needed at the position of the virtual source. Thus far we illustrated the method with numerical examples and gave heuristic arguments for its validity. Recently we presented a first mathematical derivation (Wapenaar et al., 2013). In this paper we present a more concise derivation, using a shortcut proposed by Slob and Wapenaar (2013) for the 1D situation.

\section{Green's function and fundamental solution}

Consider an inhomogeneous lossless medium below a transparent boundary $\partial \mathbb{D}_{0}$ at depth level $x_{3}=$ $x_{3,0}$. The upper half-space above $\partial \mathbb{D}_{0}$ is homogeneous. We define the spatial coordinate vector $\mathbf{x}$ as $\mathbf{x}=\left(\mathbf{x}_{\mathrm{H}}, x_{3}\right)$, in which $\mathbf{x}_{\mathrm{H}}=\left(x_{1}, x_{2}\right)$ is the horizontal coordinate vector and $x_{3}$ the vertical coordinate; the positive $x_{3}$-axis is pointing downward. Coordinates at $\partial \mathbb{D}_{0}$ are denoted as $\mathbf{x}_{0}=\left(\mathbf{x}_{\mathrm{H}}, x_{3,0}\right)$. The propagation velocity and mass density of the inhomogeneous medium are defined as $c(\mathbf{x})$ and $\rho(\mathbf{x})$, respectively. We define a second boundary $\partial \mathbb{D}_{i}$ at an arbitrary depth level $x_{3}=x_{3, i}$, with $x_{3, i}>x_{3,0}$. Coordinates at $\partial \mathbb{D}_{i}$ are denoted as $\mathbf{x}_{i}=\left(\mathbf{x}_{\mathrm{H}}, x_{3, i}\right)$. Time is denoted by $t$.

We define the Green's function $G\left(\mathbf{x}, \mathbf{x}_{0}^{\prime \prime}, t\right)$ as the solution of the scalar wave equation in the actual medium, with a source at $\mathbf{x}_{0}^{\prime \prime}$ at $t=0$, according to $\rho \nabla \cdot\left(\frac{1}{\rho} \nabla G\right)-\frac{1}{c^{2}} \frac{\partial^{2} G}{\partial t^{2}}=-\rho \delta\left(\mathbf{x}-\mathbf{x}_{0}^{\prime \prime}\right) \frac{\partial \delta(t)}{\partial t}$. Defined in this way, the Green's function is the response to an impulsive point source of volume injection rate at $\mathbf{x}_{0}^{\prime \prime}$ (de Hoop, 1995). For convenience we choose the source just above $\partial \mathbb{D}_{0}$, hence $\mathbf{x}_{0}^{\prime \prime}=\left(\mathbf{x}_{\mathrm{H}}^{\prime \prime}, x_{3,0}-\varepsilon\right)$, with $\varepsilon \rightarrow 0$. The Green's function consists of a downgoing part $G^{+}\left(\mathbf{x}, \mathbf{x}_{0}^{\prime \prime}, t\right)$ and an upgoing part $G^{-}\left(\mathbf{x}, \mathbf{x}_{0}^{\prime \prime}, t\right)$, coupled by the inhomogeneities of the medium below $\partial \mathbb{D}_{0}$ (Figure 1(a)). Throughout this paper we assume that the downgoing and upgoing fields are pressure-normalized. The vertical derivative of the downgoing part of the Green's function at $\partial \mathbb{D}_{0}$ (just below the source point) is given by

$$
\left.\partial_{3} G^{+}\left(\mathbf{x}, \mathbf{x}_{0}^{\prime \prime}, t\right)\right|_{x_{3}=x_{3,0}}=-\frac{1}{2} \rho\left(\mathbf{x}_{0}^{\prime \prime}\right) \delta\left(\mathbf{x}_{\mathrm{H}}-\mathbf{x}_{\mathrm{H}}^{\prime \prime}\right) \frac{\partial \delta(t)}{\partial t} .
$$

The vertical derivative of the upgoing part of the Green's function at $\partial \mathbb{D}_{0}$ is related to the pressurenormalized reflection response $R\left(\mathbf{x}_{0}^{\prime \prime}, \mathbf{x}_{0}, t\right)$ of the lower half-space, according to

$$
\left.\partial_{3} G^{-}\left(\mathbf{x}, \mathbf{x}_{0}^{\prime \prime}, t\right)\right|_{x_{3}=x_{3,0}}=\frac{1}{2} \rho\left(\mathbf{x}_{0}\right) \frac{\partial R\left(\mathbf{x}_{0}^{\prime \prime}, \mathbf{x}_{0}, t\right)}{\partial t} .
$$

We define a reference medium which is equal to the actual medium above $\partial \mathbb{D}_{i}$ and reflection-free below $\partial \mathbb{D}_{i}$. In this reference medium we define a fundamental solution $F(\mathbf{x}, t)$ of the source-free scalar wave equation, which consists of a downgoing part $F^{+}(\mathbf{x}, t)$ and an upgoing part $F^{-}(\mathbf{x}, t)$, coupled by the inhomogeneities of the medium between $\partial \mathbb{D}_{0}$ and $\partial \mathbb{D}_{i}$. At $\partial \mathbb{D}_{0}, F^{+}\left(\mathbf{x}_{0}, t\right)$ is shaped such that $F(\mathbf{x}, t)$ focuses at $\mathbf{x}_{i}^{\prime}=\left(\mathbf{x}_{\mathrm{H}}^{\prime}, x_{3, i}\right)$ at $t=0$, and continues as an impulsive diverging downgoing field $F^{+}(\mathbf{x}, t)$ into the reflection-free reference half-space $x_{3}>x_{3, i}$. Note that there is no upgoing field $F^{-}(\mathbf{x}, t)$ at $\partial \mathbb{D}_{i}$. The focal point $\mathbf{x}_{i}^{\prime}$ is a variable at $\partial \mathbb{D}_{i}$ and will from here onward be included in the argument list, hence $F^{( \pm)}(\mathbf{x}, t)$ becomes $F^{( \pm)}\left(\mathbf{x}, \mathbf{x}_{i}^{\prime}, t\right)$ (Figure 1(b)). The focusing condition at $\partial \mathbb{D}_{i}$ is defined, analogous to equation (1), as

$$
\left.\partial_{3} F^{+}\left(\mathbf{x}, \mathbf{x}_{i}^{\prime}, t\right)\right|_{x_{3}=x_{3, i}}=-\frac{1}{2} \rho\left(\mathbf{x}_{i}^{\prime}\right) \delta\left(\mathbf{x}_{\mathrm{H}}-\mathbf{x}_{\mathrm{H}}^{\prime}\right) \frac{\partial \delta(t)}{\partial t} .
$$

For stability reasons we exclude the major part of the evanescent wave field, hence, $\delta\left(\mathbf{x}_{\mathrm{H}}\right)$ should be interpreted as a spatially bandlimited delta function. In the next section we derive relations between the Green's functions $G^{ \pm}$and the fundamental solutions $F^{ \pm}$.

\section{Relations between the Green's function and the fundamental solution}

The Fourier transform of a time-dependent function $u(t)$ is defined as $u(\omega)=\int_{-\infty}^{\infty} u(t) \exp (-j \omega t) \mathrm{d} t$, where $\omega$ is the angular frequency and $j$ the imaginary unit. To keep the notation simple, the same symbol is used for time- and frequency-domain functions (here $u$ ). 
a)

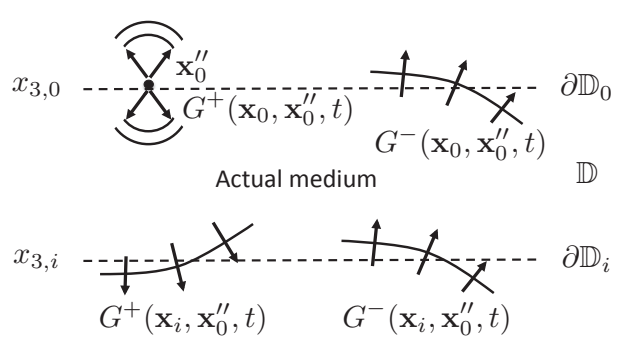

Actual medium b) Homogeneous half-space
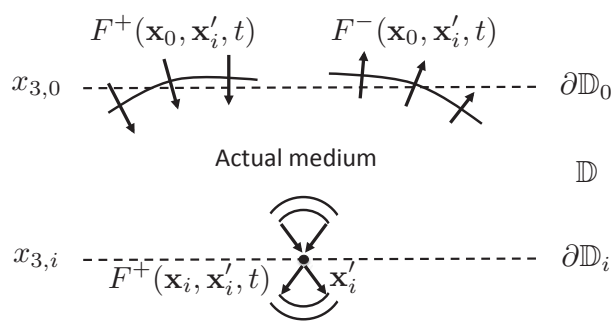

Reflection-free reference half-space

Figure 1 (a) Green's function in actual medium. (b) Fundamental solution in reference medium.

We analyze reciprocity theorems for the two independent acoustic states $F$ and $G$, defined above. We define a domain $\mathbb{D}$, enclosed by the boundaries $\partial \mathbb{D}_{0}$ and $\partial \mathbb{D}_{i}$. The domain $\mathbb{D}$ is source-free for both states and the medium parameters in both states are identical in $\mathbb{D}$ (Figure 1). For this situation the reciprocity theorems of the convolution-type and of the correlation-type read, respectively,

$$
\begin{aligned}
\int_{\partial \mathbb{D}_{0}} \rho^{-1}\left\{\left(\partial_{3} F\right) G-F\left(\partial_{3} G\right)\right\} \mathrm{d} \mathbf{x}_{0} & =\int_{\partial \mathbb{D}_{i}} \rho^{-1}\left\{\left(\partial_{3} F\right) G-F\left(\partial_{3} G\right)\right\} \mathrm{d} \mathbf{x}_{i}, \\
\int_{\partial \mathbb{D}_{0}} \rho^{-1}\left\{\left(\partial_{3} F\right)^{*} G-F^{*}\left(\partial_{3} G\right)\right\} \mathrm{d} \mathbf{x}_{0} & =\int_{\partial \mathbb{D}_{i}} \rho^{-1}\left\{\left(\partial_{3} F\right)^{*} G-F^{*}\left(\partial_{3} G\right)\right\} \mathrm{d} \mathbf{x}_{i},
\end{aligned}
$$

where the asterisk denotes complex conjugation. Substituting $F=F^{+}+F^{-}$and $G=G^{+}+G^{-}$, we find (Wapenaar and Berkhout (1989), Appendix B)

$$
\begin{aligned}
-2 \int_{\partial \mathbb{D}_{0}} \rho^{-1}\left\{F^{+}\left(\partial_{3} G^{-}\right)+F^{-}\left(\partial_{3} G^{+}\right)\right\} \mathrm{d} \mathbf{x}_{0} & =2 \int_{\partial \mathbb{D}_{i}} \rho^{-1}\left\{\left(\partial_{3} F^{+}\right) G^{-}+\left(\partial_{3} F^{-}\right) G^{+}\right\} \mathrm{d} \mathbf{x}_{i}, \\
-2 \int_{\partial \mathbb{D}_{0}} \rho^{-1}\left\{\left(F^{+}\right)^{*}\left(\partial_{3} G^{+}\right)+\left(F^{-}\right)^{*}\left(\partial_{3} G^{-}\right)\right\} \mathrm{d} \mathbf{x}_{0} & =2 \int_{\partial \mathbb{D}_{i}} \rho^{-1}\left\{\left(\partial_{3} F^{+}\right)^{*} G^{+}+\left(\partial_{3} F^{-}\right)^{*} G^{-}\right\} \mathrm{d} \mathbf{x}_{i},
\end{aligned}
$$

where in equation (7) the evanescent wave field at $\partial \mathbb{D}_{0}$ and $\partial \mathbb{D}_{i}$ is neglected. Note that at $\partial \mathbb{D}_{0}$ and $\partial \mathbb{D}_{i}$ we have chosen different combinations of the fields and their vertical derivatives (conform equations B-13 and B-34 in the mentioned reference), to make optimally use of the expressions in equations (1) (3). In the frequency domain, the time derivatives in the RHS of equations (1) - (3) are replaced by $j \omega$. Hence, substituting these expressions into equations (6) and (7) gives

$$
\begin{aligned}
\int_{\partial \mathbb{D}_{0}} F^{+}\left(\mathbf{x}_{0}, \mathbf{x}_{i}^{\prime}, \omega\right) R\left(\mathbf{x}_{0}^{\prime \prime}, \mathbf{x}_{0}, \omega\right) \mathrm{d} \mathbf{x}_{0}-F^{-}\left(\mathbf{x}_{0}^{\prime \prime}, \mathbf{x}_{i}^{\prime}, \omega\right) & =G^{-}\left(\mathbf{x}_{i}^{\prime}, \mathbf{x}_{0}^{\prime \prime}, \omega\right), \\
-\int_{\partial \mathbb{D}_{0}}\left\{F^{-}\left(\mathbf{x}_{0}, \mathbf{x}_{i}^{\prime}, \omega\right)\right\}^{*} R\left(\mathbf{x}_{0}^{\prime \prime}, \mathbf{x}_{0}, \omega\right) \mathrm{d} \mathbf{x}_{0}+\left\{F^{+}\left(\mathbf{x}_{0}^{\prime \prime}, \mathbf{x}_{i}^{\prime}, \omega\right)\right\}^{*} & =G^{+}\left(\mathbf{x}_{i}^{\prime}, \mathbf{x}_{0}^{\prime \prime}, \omega\right) .
\end{aligned}
$$

Combining these results yields

$$
G\left(\mathbf{x}_{i}^{\prime}, \mathbf{x}_{0}^{\prime \prime}, \omega\right)=\int_{\partial \mathbb{D}_{0}} F_{2}\left(\mathbf{x}_{i}^{\prime}, \mathbf{x}_{0}, \omega\right) R\left(\mathbf{x}_{0}^{\prime \prime}, \mathbf{x}_{0}, \omega\right) \mathrm{d} \mathbf{x}_{0}+\left\{F_{2}\left(\mathbf{x}_{i}^{\prime}, \mathbf{x}_{0}^{\prime \prime}, \omega\right)\right\}^{*},
$$

with $F_{2}\left(\mathbf{x}_{i}^{\prime}, \mathbf{x}_{0}, \omega\right)=F^{+}\left(\mathbf{x}_{0}, \mathbf{x}_{i}^{\prime}, \omega\right)-\left\{F^{-}\left(\mathbf{x}_{0}, \mathbf{x}_{i}^{\prime}, \omega\right)\right\}^{*}$. It can be shown that $F_{2}\left(\mathbf{x}_{i}^{\prime}, \mathbf{x}_{0}, \omega\right)$ is another fundamental solution that focuses at $\mathbf{x}_{0}$ and continues as a diverging upgoing field into the homogeneous upper half-space $x_{3}<x_{3,0}$ (Wapenaar et al., 2013). In the next section we use equation (10) as a basis for deriving the 3D Marchenko equation.

\section{D Marchenko equation}

We transform equation (10) to the time domain, which gives

$$
G\left(\mathbf{x}_{i}^{\prime}, \mathbf{x}_{0}^{\prime \prime}, t\right)=\int_{\partial \mathbb{D}_{0}} \mathrm{~d} \mathbf{x}_{0} \int_{-\infty}^{\infty} F_{2}\left(\mathbf{x}_{i}^{\prime}, \mathbf{x}_{0}, t^{\prime}\right) R\left(\mathbf{x}_{0}^{\prime \prime}, \mathbf{x}_{0}, t-t^{\prime}\right) \mathrm{d} t^{\prime}+F_{2}\left(\mathbf{x}_{i}^{\prime}, \mathbf{x}_{0}^{\prime \prime},-t\right) .
$$


Let $t_{\mathrm{d}}\left(\mathbf{x}_{i}^{\prime}, \mathbf{x}_{0}^{\prime \prime}\right)$ denote the traveltime of the first arrival between $\mathbf{x}_{0}^{\prime \prime}$ just above $\partial \mathbb{D}_{0}$ and $\mathbf{x}_{i}^{\prime}$ at $\partial \mathbb{D}_{i}$. By evaluating equation (11) only for $t<t_{\mathrm{d}}\left(\mathbf{x}_{i}^{\prime}, \mathbf{x}_{0}^{\prime \prime}\right)$, the left-hand side can be replaced by zero. Equation (11) (with 0 on the left-hand side) only constrains $F_{2}$ up to a multiplicative constant and therefore an ansatz will be made for the form of this function. Analogous to the 1D situation (Lamb, 1980), the ansatz for $F_{2}\left(\mathbf{x}_{i}^{\prime}, \mathbf{x}_{0}, t\right)$ is a superposition of a direct wave and a coda of multiple scattered arrivals, according to

$$
F_{2}\left(\mathbf{x}_{i}^{\prime}, \mathbf{x}_{0}, t\right)=G_{\mathrm{d}}\left(\mathbf{x}_{i}^{\prime}, \mathbf{x}_{0},-t\right)+\theta\left(t+t_{\mathrm{d}}\left(\mathbf{x}_{i}^{\prime}, \mathbf{x}_{0}\right)\right) M\left(\mathbf{x}_{i}^{\prime}, \mathbf{x}_{0}, t\right) .
$$

Here $G_{\mathrm{d}}\left(\mathbf{x}_{i}^{\prime}, \mathbf{x}_{0},-t\right)$ is the time-reversed direct arrival of the Green's function; its arrival time is $-t_{\mathrm{d}}\left(\mathbf{x}_{i}^{\prime}, \mathbf{x}_{0}\right)$ (a more accurate approach for defining the direct wave in equation (12) is based on an inverse of the vertical derivative of a transmission operator, but its discussion is beyond the scope of this paper). $M\left(\mathbf{x}_{i}^{\prime}, \mathbf{x}_{0}, t\right)$ is the coda following the direct arrival. It is the result of scattering taking place in the inhomogeneous medium in $\mathbb{D}$. The coda is assumed to be causal, i.e., $M\left(\mathbf{x}_{i}^{\prime}, \mathbf{x}_{0}, t\right)=0$ for $t<-t_{\mathrm{d}}\left(\mathbf{x}_{i}^{\prime}, \mathbf{x}_{0}\right)$. This causality is expressed by multiplying the coda with the Heaviside function $\theta\left(t+t_{\mathrm{d}}\left(\mathbf{x}_{i}^{\prime}, \mathbf{x}_{0}\right)\right)$ in equation (12). Note that the ansatz limits the validity of what follows to configurations for which the ansatz holds true. For example, it holds in layered media with moderately curved interfaces as long as $\left|\mathbf{x}_{\mathrm{H}}^{\prime}-\mathbf{x}_{\mathrm{H}}\right|$ is not too large (to avoid occurence of turning waves, headwaves etc.). The conditions underlying the ansatz need further investigation, which is beyond the scope of this paper. Substituting equation (12) into (11) (with 0 on the left-hand side) yields

$$
\begin{aligned}
0 & =\int_{\partial \mathbb{D}_{0}} \mathrm{~d} \mathbf{x}_{0} \int_{-\infty}^{\infty} G_{\mathrm{d}}\left(\mathbf{x}_{i}^{\prime}, \mathbf{x}_{0},-t^{\prime}\right) R\left(\mathbf{x}_{0}^{\prime \prime}, \mathbf{x}_{0}, t-t^{\prime}\right) \mathrm{d} t^{\prime} \\
& +\int_{\partial \mathbb{D}_{0}} \mathrm{~d} \mathbf{x}_{0} \int_{-t_{\mathrm{d}}\left(\mathbf{x}_{i}^{\prime} \mathbf{x}_{0}\right)}^{\infty} M\left(\mathbf{x}_{i}^{\prime}, \mathbf{x}_{0}, t^{\prime}\right) R\left(\mathbf{x}_{0}^{\prime \prime}, \mathbf{x}_{0}, t-t^{\prime}\right) \mathrm{d} t^{\prime}+M\left(\mathbf{x}_{i}^{\prime}, \mathbf{x}_{0}^{\prime \prime},-t\right),
\end{aligned}
$$

which holds for $t<t_{\mathrm{d}}\left(\mathbf{x}_{i}^{\prime}, \mathbf{x}_{0}^{\prime \prime}\right)$ because of causality. This is the 3D single-sided Marchenko equation. Next we show that it can be solved for $M\left(\mathbf{x}_{i}^{\prime}, \mathbf{x}_{0}, t\right)$ with an iterative 3D data-driven focusing scheme.

\section{D data-driven focusing and Green's function retrieval}

Inspired by the work of Rose (2001), we proposed an iterative scheme to design a downgoing wave field $p^{+}\left(\mathbf{x}_{0}, t\right)$ at $\partial \mathbb{D}_{0}$ that focuses at $t=0$ at a designated focal point $\mathbf{x}_{\mathrm{F}}$ (and at $\mathbf{x}_{\mathrm{F}}$ only) in the actual medium below $\partial \mathbb{D}_{0}$ (Wapenaar et al., 2012a). The initial estimate $p_{0}^{+}\left(\mathbf{x}_{0}, t\right)$ is defined as $p_{0}^{+}\left(\mathbf{x}_{0}, t\right)=$ $G_{\mathrm{d}}\left(\mathbf{x}_{0}, \mathbf{x}_{\mathrm{F}},-t\right)$. When emitted from $\partial \mathbb{D}_{0}$ into the inhomogeneous medium, this field not only focuses at $\mathbf{x}_{\mathrm{F}}$, but it also causes ghost images at $t=0$. These ghost images can be cancelled by updating the incident field, which causes new ghost images, which again need to be cancelled, etc. The following iterative scheme accomplishes this task (Wapenaar et al., 2012a)

$$
\begin{aligned}
& p_{k}^{+}\left(\mathbf{x}_{0}, \mathbf{x}_{\mathrm{F}}, t\right)=G_{\mathrm{d}}\left(\mathbf{x}_{0}, \mathbf{x}_{\mathrm{F}},-t\right)-\theta\left(t+t_{\mathrm{d}}\left(\mathbf{x}_{0}, \mathbf{x}_{\mathrm{F}}\right)\right) p_{k-1}^{-}\left(\mathbf{x}_{0}, \mathbf{x}_{\mathrm{F}},-t\right), \\
& p_{k}^{-}\left(\mathbf{x}_{0}^{\prime \prime}, \mathbf{x}_{\mathrm{F}}, t\right)=\int_{\partial \mathbb{D}_{0}} \mathrm{~d} \mathbf{x}_{0} \int_{-\infty}^{\infty} R\left(\mathbf{x}_{0}^{\prime \prime}, \mathbf{x}_{0}, t-t^{\prime}\right) p_{k}^{+}\left(\mathbf{x}_{0}, \mathbf{x}_{\mathrm{F}}, t^{\prime}\right) \mathrm{d} t^{\prime} .
\end{aligned}
$$

Here $p_{k}^{+}\left(\mathbf{x}_{0}, \mathbf{x}_{\mathrm{F}}, t\right)$ is the $k$ th iteration of the downgoing wave field, intended to focus at $\mathbf{x}_{\mathrm{F}}$, whereas $p_{k}^{-}\left(\mathbf{x}_{0}^{\prime \prime}, \mathbf{x}_{\mathrm{F}}, t\right)$ is its upgoing reflection response. The scheme starts for $k=0$, with $p_{-1}^{-}\left(\mathbf{x}_{0}, \mathbf{x}_{\mathrm{F}},-t\right)=0$. Unlike the 1D scheme of Rose, which only needs the reflection response as input, this 3D scheme requires the reflection response $R\left(\mathbf{x}_{0}^{\prime \prime}, \mathbf{x}_{0}, t\right)$ and the direct Green's function $G_{\mathrm{d}}\left(\mathbf{x}_{0}, \mathbf{x}_{\mathrm{F}}, t\right)$. The reflection response is obtained from measurements at the boundary $\partial \mathbb{D}_{0}$. The direct Green's function mainly requires an estimate of the direct arrival time $t_{\mathrm{d}}\left(\mathbf{x}_{0}, \mathbf{x}_{\mathrm{F}}\right)$, for which no detailed information about the medium is required: a smooth background model suffices to compute the direct Green's function $G_{\mathrm{d}}\left(\mathbf{x}_{0}, \mathbf{x}_{\mathrm{F}}, t\right)$. All information about the scattering properties of the medium comes from the measured reflection response. This is why we call the scheme of equations (14) and (15) "data-driven focusing". Assuming the scheme converges, the final result is denoted by dropping the subscripts $k-1$ and $k$. Substituting equation (14) into equation (15), using source-receiver reciprocity for the direct Green's function, choosing $\mathbf{x}_{\mathrm{F}}$ at $\partial \mathbb{D}_{i}$, gives for $t<t_{\mathrm{d}}\left(\mathbf{x}_{\mathrm{F}}, \mathbf{x}_{0}^{\prime \prime}\right)$ the 3D Marchenko equation (13), with $M\left(\mathbf{x}_{\mathrm{F}}, \mathbf{x}_{0}^{\prime \prime},-t\right)=-p^{-}\left(\mathbf{x}_{0}^{\prime \prime}, \mathbf{x}_{\mathrm{F}}, t\right)$. Hence, 

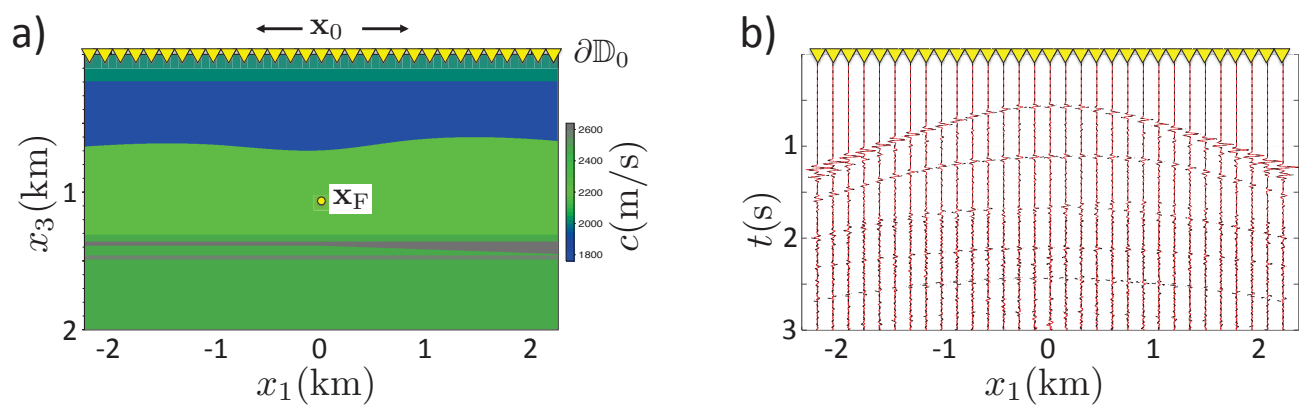

Figure 2 (a) Subsurface model. (b) Retrieved Green's function (black) and reference solution (red).

the iterative 3D data-driven focusing scheme of equations (14) and (15) solves the 3D Marchenko equation. Next, observe that comparison of equation (12) with equation (14) (without the subscripts $k-1$ and $k$ ) gives $F_{2}\left(\mathbf{x}_{\mathrm{F}}, \mathbf{x}_{0}, t\right)=p^{+}\left(\mathbf{x}_{0}, \mathbf{x}_{\mathrm{F}}, t\right)$. Substituting this into equation (11), using equation (15) and source-receiver reciprocity for the Green's function, gives (dropping the double primes)

$$
G\left(\mathbf{x}_{0}, \mathbf{x}_{\mathrm{F}}, t\right)=p^{+}\left(\mathbf{x}_{0}, \mathbf{x}_{\mathrm{F}},-t\right)+p^{-}\left(\mathbf{x}_{0}, \mathbf{x}_{\mathrm{F}}, t\right) .
$$

This shows that a combination of the focusing wave field $p^{+}$and its response $p^{-}$yields the Green's function with its virtual source at the position of the focal point inside the medium. Unlike in other Green's function retrieval methods, no physical receiver is required at the position of the virtual source. The method is illustrated with a numerical example in Figure 2. A more challenging example is discussed in a companion paper (Broggini et al., 2013).

\section{Conclusions}

We have derived a 3D version of the Marchenko equation, which relates the single-sided 3D reflection response of an inhomogeneous medium to a field inside the medium. In the derivation we assumed scalar waves in a lossless medium, ignored evanescent waves and further assumed that the scattering coda of the fundamental solution follows the direct arrival of the time-reversed Green's function. These conditions imply some restrictions that need further investigation. For those situations in which the conditions are fulfilled, we showed that the 3D single-sided Marchenko equation is solved by our iterative data-driven focusing scheme. This scheme requires the $3 \mathrm{D}$ reflection response at one side of the medium and an estimate of the direct arrival of the Green's function. We also showed that a combination of the focusing wave field and its reflection response gives the 3D Green's function with its virtual source located at the focal point. Because no physical receiver is needed at the position of the focal point, the focal point can be chosen anywhere inside the medium. This gives the possibility to obtain Green's functions with virtual sources throughout the medium, which can be used for integrated migration and internal multiple elimination (Wapenaar et al., 2012b). The next challenge is to extend the method to vector wave fields.

\section{References}

Broggini, F. and Snieder, R. [2012] Connection of scattering principles: a visual and mathematical tour. Eur. J. Phys., 33, 593-613.

Broggini, F., Snieder, R., Wapenaar, K. and Thorbecke, J. [2013] Creating the Green's response to a virtual source inside a medium using reflection data with internal multiples. EAGE, Extended Abstracts, (submitted).

de Hoop, A.T. [1995] Handbook of radiation and scattering of waves. Academic Press, London.

Lamb, G.L. [1980] Elements of soliton theory. John Wiley and Sons, Inc., New York.

Rose, J.H. [2001] "Single-sided" focusing of the time-dependent Schrödinger equation. Phys. Rev. A, $65,012707$.

Slob, E. and Wapenaar, K. [2013] GPR wave field decomposition, synthesis and imaging for lossless layered VTI media. 7th International Workshop on Advanced Ground Penetrating Radar, (submitted).

Wapenaar, C.P.A. and Berkhout, A.J. [1989] Elastic wave field extrapolation. Elsevier, Amsterdam.

Wapenaar, K., Broggini, F., Slob, E. and Snieder, R. [2013] Three-dimensional single-sided Marchenko inverse scattering, data-driven focusing, Green's function retrieval, and their mutual relations. Phys. Rev. Lett., (subm).

Wapenaar, K., Broggini, F. and Snieder, R. [2012a] Creating a virtual source inside a medium from reflection data: heuristic derivation and stationary-phase analysis. Geophys. J. Int., 190, 1020-1024.

Wapenaar, K. et al. [2012b] Integrated migration and internal multiple elimination. SEG, Expanded Abstracts, SPMUL 1.5). 